\title{
ВмJ Global Health International research and guidelines on post-tuberculosis chronic lung disorders: a systematic scoping review
}

\author{
Sanne C van Kampen, ${ }^{1}$ Amanda Wanner, ${ }^{1}$ Miles Edwards, ${ }^{1}$ Anthony D Harries, ${ }^{2,3}$ \\ Bruce J Kirenga, ${ }^{4}$ Jeremiah Chakaya, ${ }^{2,5}$ Rupert Jones ${ }^{1}$
}

To cite: van Kampen SC, Wanner A, Edwards M, et al. International research and guidelines on posttuberculosis chronic lung disorders: a systematic scoping review. BMJ Glob Health 2018;3:e000745. doi:10.1136/ bmjgh-2018-000745

Handling editor Alberto $L$ Garcia-Basteiro

- Additional material is published online only. To view please visit the journal online (http://dx.doi.org/10.1136/ bmjgh-2018-000745).

Received 30 January 2018 Revised 18 May 2018 Accepted 28 May 2018
Check for updates

\section{Author(s) (or their} employer(s)) 2018. Re-use permitted under CC BY-NC. No commercial re-use. See rights and permissions. Published by BMJ.

For numbered affiliations see end of article.

Correspondence to Dr Sanne C van Kampen; sanne.vankampen@gmail.com

\section{ABSTRACT}

Introduction Pulmonary tuberculosis (TB) is an important risk factor for chronic respiratory disease due to residual lung damage. Yet, the WHO End TB strategy does not mention post-TB chronic lung disorders (PTBLDs) and programmatic interventions to address PTBLD are lacking. This study assessed the scope of current guidelines and evidence on PTBLD to inform policy and research action. Methods A systematic literature search was conducted following Preferred Reporting Items for Systematic Review and Meta-Analysis guidelines. Eight databases (TRIP, International Guideline Library, MEDLINE/PubMed, EMBASE, Web of Science, Global Health, Cochrane Library) were searched for records on PTBLD published between 1 January 1990 and 1 December 2017. Non-English records, case series, conference abstracts and letters to editors were excluded. Data were extracted and charted on publication year, location, PTBLD condition(s) and main study outcome.

Results A total of 212 guidelines and 3661 articles were retrieved. After screening, only three international TB guidelines mentioned TB sequelae, but none described how to identify or manage the condition. A total of 156 articles addressed PTBLD: 54 (35\%) mentioned unspecified TB sequelae; 47 (30\%) specific post-TB conditions including aspergillosis, bronchial stenosis or bronchiectasis; 52 (33\%) post-TB obstructive disorders or lung function impairment; and 20 (13\%) post-TB respiratory symptoms or chest $\mathrm{X}$-ray abnormalities. The first two groups mostly assessed surgery or ventilation techniques for patient management, while the last two groups typically assessed prevalence or predictors of disease.

Conclusion This is the first review to provide a comprehensive overview of the current literature on PTBLD. The scope of evidence around the burden of PTBLD warrants inclusion and recognition of the problem in international TB guidelines. Research is now needed on early detection of PTBLD and patient management options that are suitable for high-burden TB countries.

\section{INTRODUCTION}

The burden of chronic respiratory disease is growing worldwide with chronic obstructive pulmonary disease (COPD) now being the third leading cause of death. ${ }^{1}$ An estimated

\section{Key questions}

What is already known?

- Pulmonary tuberculosis (TB) is an important risk factor for chronic respiratory disease due to residual lung damage.

- The scope of current guidelines and evidence on post-TB chronic lung disorders (PTBLDs) is unknown.

What are the new findings?

- Out of 212 international TB guidelines, only three mentioned TB sequelae and none described how to identify or manage the condition.

- Of 156 scientific articles on PTBLD, around twothirds addressed treatment by surgery or ventilation techniques and one-third addressed prevalence or predictors of the condition.

What do the new findings imply?

- While the scope of evidence on the burden of PTBLD justifies inclusion in international guidelines, more research is needed on patient management options that are suitable for high-burden TB countries.

3.2 million people died of COPD globally in 2015; an increase of $12 \%$ compared with $1990 .^{2}$ The main known risk factors for chronic respiratory diseases are smoking and outdoor air pollution, followed by household smoke exposure, occupational dust exposure, ozone and second-hand smoke. ${ }^{3}$ In recent years, evidence has emerged that tuberculosis (TB) is also an important predictor of COPD. Systematic reviews and international surveys have shown that a history of TB can increase the odds of COPD by an average of threefold and even more in countries with a high burden of TB. ${ }^{4-8}$ Pulmonary TB can lead to irreversible lung damage visible as scarring, fibrosis, cavitation or other types of damage on radiological images. This in turn can lead to loss of lung function, long-term respiratory symptoms and eventually chronic respiratory disease, including COPD, bronchiectasis and aspergillosis. ${ }^{9-13}$ These conditions can 
jointly been referred to as post-TB chronic lung disorders (PTBLDs). ${ }^{14}$ As TB disease remains one of the top 10 causes of mortality worldwide with an estimated 10.4 million new cases and 1.3 million deaths in 2016, the problem of PTBLD could be substantial. ${ }^{15}$

The latest WHO Global Action Plan on Non-communicable Diseases has recognised the strong interaction between TB and chronic respiratory diseases, particular in low-income and middle-income countries. ${ }^{16}$ On the other hand, the WHO End TB strategy and the Sustainable Development Goals (SDG 3.3) still focus on reducing $\mathrm{TB}$ incidence and $\mathrm{TB}$ mortality and do not mention PTBLD. ${ }^{17}{ }^{18}$ Current WHO-recommended TB registries only capture mortality and morbidity during TB treatment, and as a result, patients are not followed up beyond cure of disease. ${ }^{19}$ Programmatic interventions to monitor ex-TB patients and address PTBLD are also lacking. ${ }^{20}{ }^{21}$ This raises the question whether the scope of current evidence around PTBLD is robust enough to inform immediate policy and research action. This study set out to identify all international guidelines and scientific articles on PTBLD and map them in terms of main conditions and outcomes under study as well as their geographical and temporal spread.

\section{METHODS}

\section{Search strategy and selection criteria}

A systematic review was conducted following the current Preferred Reporting Items for Systematic Review and Meta-Analysis guidelines. ${ }^{22} 23$ On 29 June 2017, we searched the TRIP medical database and International Guideline Library for international guidelines using the search term 'tuberculosis' and limiting by 'guidelines' and 'English language'. ${ }^{24}{ }^{25}$ Titles of the international guidelines were screened and those addressing TB and patient management were kept, excluding those focusing on prevention, screening, diagnosis or latent infection. The full text of included guidelines was screened for inclusion of the words: 'post-tuberculosis', 'post-TB', 'sequelae', 'destroyed lung', 'after/following cure', 'healed', 'after/following treatment', 'follow up'; and excluded if they did not address PTBLD.

We searched the following databases for peer-reviewed articles: MEDLINE, PubMed, EMBASE, Web of Science, Global Health and the Cochrane Library. The search strategy was iteratively developed and run by an information specialist, using search terms for 'post-tuberculosis' and 'chronic respiratory disorders' and was limited to records published between 1 January 1990 and 1 December 2017. A combination of free text and controlled vocabulary terms were used as appropriate and translated for use in each database. The citations were downloaded and managed in EndNote X8. An example of the search strategy used in Ovid MEDLINE is shown in online supplementary file 1 . Two reviewers screened the titles and abstracts using an online toolRayyan. ${ }^{26}$ A pilot screening of 220 abstracts was done by three authors to help clarify the inclusion and exclusion criteria and ensure disagreement was less than 5\%. Two reviewers screened all abstracts and included studies that had a human population with a history of TB disease, and that assessed a range of conditions that could potentially be caused by past TB disease. We excluded studies that primary focused on a specific lung condition and assessed people with a history of TB only as a subpopulation without reporting main study outcomes. We also excluded non-English records, case series, conference abstracts or letters to editors. The specific inclusion and exclusion criteria for screening are listed in online supplementary file 2 .

\section{Data analysis}

For guidelines, data were extracted on author, publication year, title, location and type of PTBLD condition being addressed. Text from the guidelines that made any reference to PTBLD was quoted. For articles, data were extracted on first author, publication year, study location, type of PTBLD condition and main study outcome. A pilot data extraction of 10 papers was done by three reviewers to clarify definitions. Full data extraction was performed by two reviewers who double-read the abstracts and full texts if required.

Following Arksey and O'Malley's framework for scoping reviews, ${ }^{23}$ the reviewers added labels to the articles for each type of PTBLD condition under study: TB sequelae (unspecified), post-TB aspergillosis, bronchiectasis, haemoptysis, bronchial stenosis, obstructive disease, pulmonary function impairment, radiological lung abnormalities and/or respiratory symptoms. Each study could receive one or more labels for condition under study, as studies could assess, for example, post-TB respiratory symptoms as well as chest X-ray abnormalities. Reviewers further added a label to the articles for main outcome under study: prevalence (population-based), proportion (limited study population), predictors/risk factors, comorbidities/complications, progression (longitudinal), management (pulmonary surgery, ventilation, rehabilitation, medication) or prevention. Each study received only one label presenting the most important outcome of the study. Online supplementary file 3 shows the definitions used for the main study outcomes. Using these labels for conditions and outcome, the studies were cross-tabulated in an evidence map. Publication year was grouped into 5-year brackets from 1990 onwards. Country of study was grouped into geographical similar regions. Multiregional studies and literature reviews without a geographical restriction were grouped as 'global'. Graphs were used to visualise periodical and geographical spread. Disagreement between the two reviewers was solved by discussion with a third reviewer.

We did not perform quality assessments of the included studies because our review aimed to map all available evidence and not to summarise the results of the studies. Critical appraisal is not normally a part of the scoping review process since by intention, studies of highly varying 


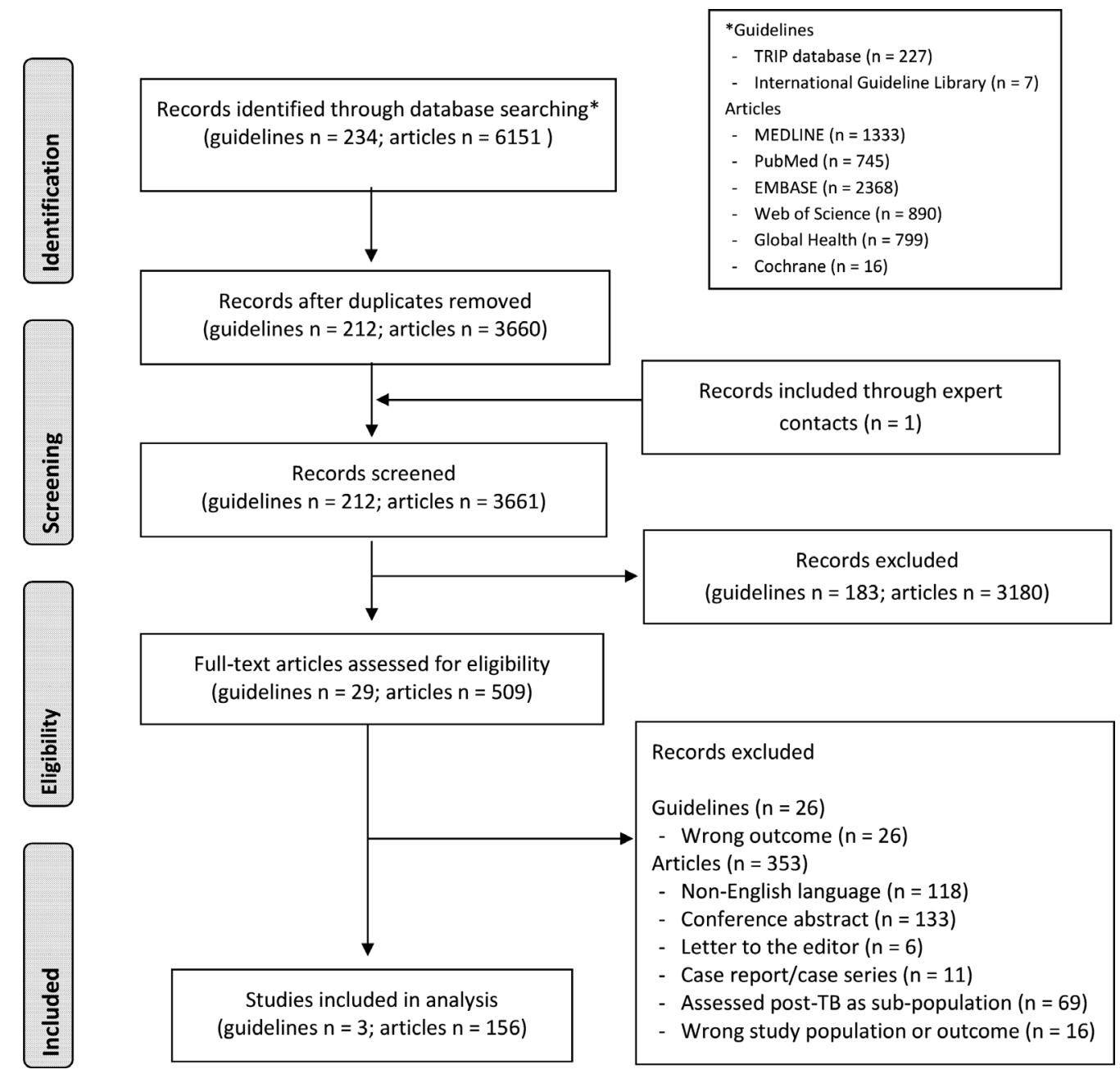

Figure 1 Flow diagram of guidelines and articles on post-tuberculosis (TB) chronic lung disorders retrieved and included in the review.

designs and methodologies are included for which no single quality assessment tool is available. As a compromise, we highlighted and discussed all systematic reviews found through our search.

\section{RESULTS}

After removal of duplicates, 212 international guidelines and 3661 peer-reviewed articles were retrieved and screened for relevance, after which 29 guidelines and 509 articles were included in full-text screening (figure 1). After full-text screening, only three guidelines and 156 articles adhered to the inclusion criteria and were included in the analysis.

A total of seven international TB guidelines addressed patient follow-up after TB treatment. However, only three actually mentioned the problem of TB sequelae, while the other four referred to the risk of relapse or recurrence of TB disease. ${ }^{27-30}$ As quoted in box 1, one international guideline indicated that early diagnosis of TB may lead to fewer TB sequelae. ${ }^{31}$ Two others eluded to the problem of long-term TB sequelae and were both written for low-burden TB countries. While one guideline expressed the need for post-cure management and support to address TB sequelae, the other indicated no need for clinical monitoring after cure of TB. ${ }^{32}{ }^{33}$ None of these guidelines described how potential TB sequelae should be identified or managed.

Of the 509 articles included for full-text screening, 353 were excluded based on the exclusion criteria. Online supplementary file 4 shows a list of the 156 final articles and their characteristics. Table 1 shows an evidence map of the number of studies by main post-TB conditions and outcome under study. Among the 156 included studies, $54(35 \%)$ evaluated unspecified TB sequelae, 47 (30\%) evaluated various types of specific post-TB disorders (aspergillosis, bronchiectasis, haemoptysis, bronchial stenosis, pulmonary hypertension, emphysema, fibrosis, asthma), 52 (33\%) evaluated post-TB obstructive disorders and/or pulmonary function impairments, and 20 (13\%) evaluated post-TB radiological abnormalities and/or respiratory symptoms.

Figure 2 shows the number of studies by publication period and geographic location for different groups of post-TB conditions. Half the studies on unspecified TB sequelae assessed pulmonary surgery or ventilation techniques to manage patients $(27 / 54)$. Ten studies looked at 
Box 1 Quotes from international guidelines on tuberculosis (TB) that addressed post-TB chronic lung disorders

International Union Against Tuberculosis and Lung Diseases, France 2003. Tuberculosis guide for specialist physicians. Page 70. "Early diagnosis affords a double benefit: the affected individual undergoes earlier treatment, which results in fewer sequelae and improved survival, and the period during which the individual is able to spread the infection in the community is shortened".

Ministry of Science and Innovation, Spain 2010. Clinical practice guidelines on the diagnosis, treatment and prevention of tuberculosis.

Page 26. "In most patients, clinical monitoring is not recommended after treatment has been correctly completed".

Page 33. "Chronic pulmonary sequelae after acute infection has been cured are responsible for most of the deterioration in patients' quality of life".

World Health Organization, Switzerland 2014. Towards TB elimination; action framework for low-incidence countries. Pages 37 and 65: "Treatment and support must extend beyond cure to address any sequelae associated with TB. Access to comprehensive palliative and end-of-life care is essential, particularly for people with M/XDR-TB".

comorbidities/complications of TB sequelae, including lung function (5), chest X-ray abnormalities (4) and dyspnoea (3). Seven studies looked at disease progression of which four focused on mortality. Five studies assessed pulmonary rehabilitation. Most studies did not specify how people with TB sequelae were defined, identified or diagnosed. Three studies referred to TB sequelae as 'post-TB lung disorder/disease' and six as 'TB destroyed lung'. The latter commonly refers to lung tissue scarring visible on chest X-ray. Studies on TB sequelae have been published since 1990 and have mostly come from the USA, Europe, South Korea, Japan and Taiwan, ie, from countries with a relatively low burden of TB.

Since 2010, there has been an increase in studies on specific post-TB conditions, including aspergillosis, bronchiectasis, bronchial stenosis, haemoptysis, pulmonary hypertension, emphysema, fibrosis and asthma. These studies have mostly been conducted in South Korea, Japan, Taiwan, India and Pakistan. Studies on post-TB aspergillosis primarily addressed population-based prevalence $(7 / 13)$, while studies on bronchial stenosis and haemoptysis focused on pulmonary surgery $(9 / 9 ; 5 / 5)$. One systematic review assessed the proportion of patients with bronchiectasis and found that people with a history of TB had higher odds of this condition (based on two studies with ORs of 3.07 (95\% CI 1.89 to 4.98 ) and 4.61 (95\% CI 2.39 to 8.88 ), respectively). ${ }^{6}$

The majority of studies on post-TB lung function impairment or obstructive disorders measured the proportion $(25 / 52)$, predictors $(12 / 52)$ or prevalence $(6 / 52)$ of these conditions. Two systematic reviews assessed the proportion of COPD among people with a history of TB disease ${ }^{67}$ One reported a global pooled OR of 3.05 (95\% CI 2.42 to 3.85) and the other found ORs ranging between 1.37 and 2.94. ORs were correlated with national TB incidence rates. ${ }^{6}$ There has been a considerable increase in the number of studies on post-TB lung function impairment and obstructive disease since 2010 and they have been conducted in most regions of the world. However, limited studies have assessed comorbidities or progression and none assessed patient management options.

Most studies on post-TB chest X-ray abnormalities and respiratory symptoms were conducted in India and Pakistan and almost exclusively reported on the proportion of these conditions (16/20). One systematic review assessed pulmonary abnormalities with radiological imaging among people with a history of $\mathrm{TB}$ and found proportions of between $8.3 \%-83.7 \%$ for cavitation, $4.3 \%-11.2 \%$ for bronchiectasis and $25.0 \%-70.4 \%$ for fibrosis. ${ }^{13}$ Studies on other outcomes, including prevalence and predictors, of post-TB chest X-ray abnormalities and respiratory symptoms were lacking.

\section{DISCUSSION}

This review found that international TB guidelines universally fail to address the importance of PTBLD, particularly omitting guidance on identification and management. This is alarming given the global rise in mortality and morbidity from non-communicable diseases, including chronic respiratory diseases. ${ }^{1418}$ Consequently, national TB policies do not address the issue and national TB control programmes are not designed to adequately deal with the problem. Since the focus is on identifying and treating active $\mathrm{TB}$ disease, patients with $\mathrm{TB}$ are usually sent home after bacteriological confirmation of cure at the end of treatment. In case they continue to or return with a cough, recurrent TB or drug-resistant TB may be expected even in the absence of positive laboratory results. It is likely, however, that a proportion of smear-negative recurrent $\mathrm{TB}$ is in fact unrecognised chronic lung disease. In resource-limited countries where there are limited facilities for diagnosing chronic lung disease, some of these patients are probably commenced on re-treatment anti-TB regimens, which are unnecessary and potentially harmful in terms of toxicity. Also, in many countries, people with continuous respiratory symptoms after TB cure may be believed to be infectious and can be stigmatised. ${ }^{34}$ Greater awareness of PTBLD among policy-makers, practitioners and patients is therefore warranted.

While TB guidelines fail to address PTBLD, this review found an abundance of studies on the prevalence and proportion of PTBLD. Retrieved systematic reviews confirm that a history of TB is an important risk factor for COPD, especially in high-burden TB countries. ${ }^{14}$ On the other hand, there was a lack of studies on progression and patient management of PTBLD (besides surgery), which may partly explain the absence of recommendations on 


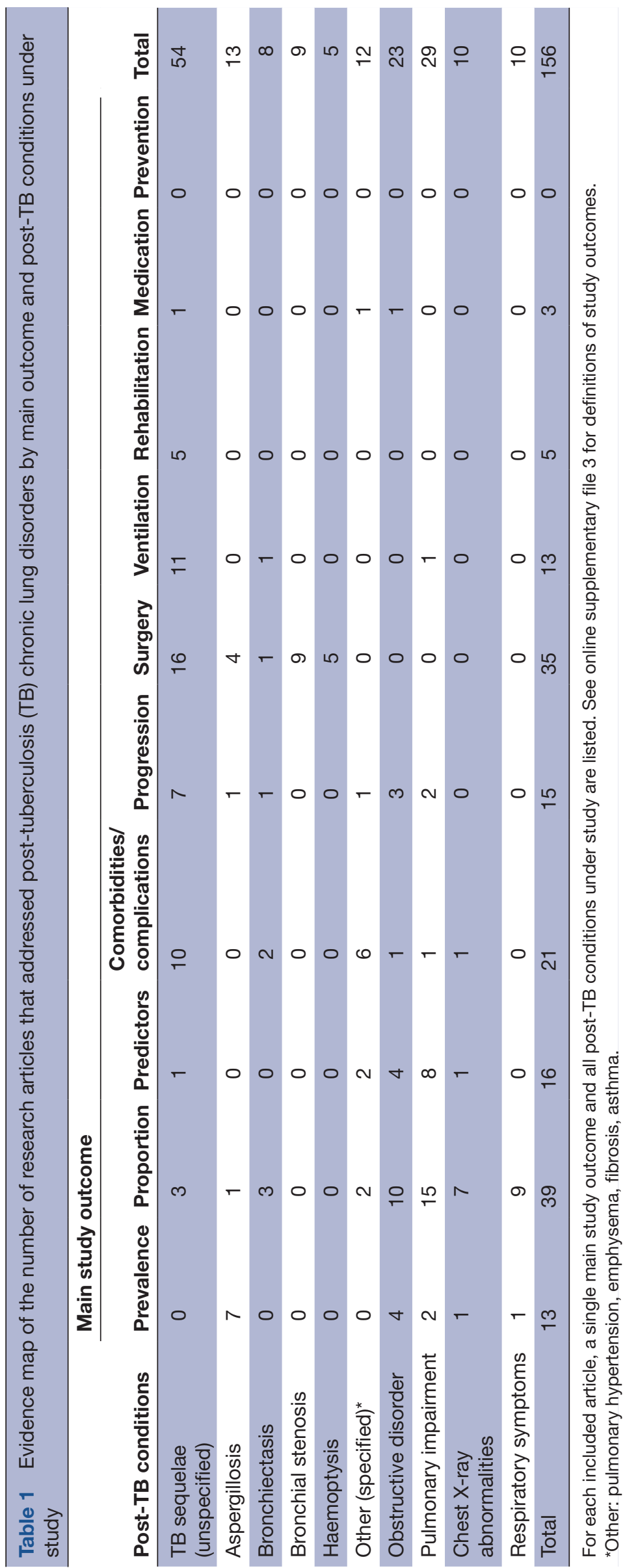

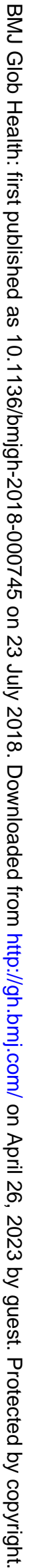



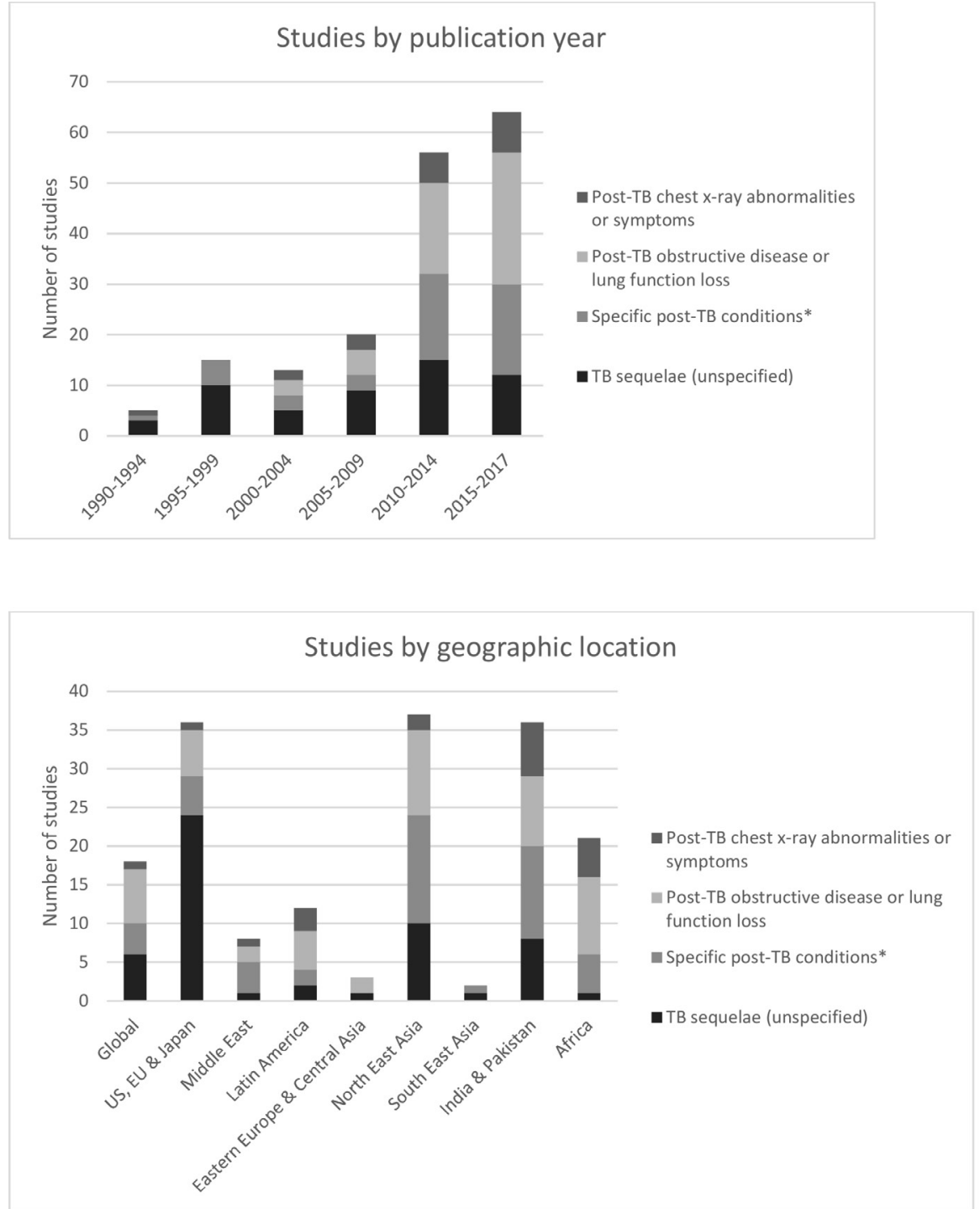

*Aspergillosis, bronchiectasis, haemoptysis, bronchial stenosis, pulmonary hypertension, emphysema, fibrosis, asthma

Figure 2 Periodical and geographical distribution of research articles on post-tuberculosis (TB) chronic lung disorders.

post-TB follow-up. The scope of evidence on the burden of PTBLD should be sufficient to justify inclusion in TB guidelines. As a minimum, healthcare staff should be made aware of the increased risk that ex-TB patients face in developing chronic respiratory disorders, so that they can discuss the health implications of residual lung damage with patients and their families. Ideally, those at risk should be referred to chronic lung disease services as early as possible to receive appropriate diagnosis (with, eg, spirometry) and treatment (with, eg, inhalers) in the same way that people with COPD and similar conditions would.

Unfortunately, the mechanisms of early referral are yet to be assessed and determined and, importantly, chronic lung disease services are often not yet established in highburden TB countries. ${ }^{16}$ Since there is no agreed way to follow-up ex-TB patients, people with PTBLD are likely to either suffer in silence or report to a health facility in a very late and severe state of disease. It could be sensible to offer symptom assessment, chest imaging and lung function tests to ex-TB patients immediately or a few months after their treatment completion while they are still on the registries. ${ }^{35-37}$ Obviously, these services should be made available to all people with chronic respiratory disease. There may also be other early predictors of disease including certain biomarkers. An international study called TB Sequel has been initiated in Gambia, Tanzania, Mozambique and South Africa to assess predictors and progression of PTBLD among a cohort of patients with $\mathrm{TB}$, which could provide valuable information on how to best follow-up ex-TB patients. ${ }^{38}$ With regards to patient management, there is a desperate need to establish services for all chronic lung disorders and for more studies to examine care options appropriate to low resource settings. Whereas surgery, ventilation techniques, medication and behaviour change interventions such as smoking cessation may be useful in countries where TB burdens are usually low, general education on 
lung health may be more suitable for high-burden countries and pulmonary rehabilitation offers a new way to improve health status in PTBLD. ${ }^{39}$ While limiting our search to English language studies could have introduced bias, over $80 \%$ of excluded articles came from Japan and Europe and would not have affected these conclusions.

This review has revealed a potential problem with the definition of PTBLD. It was surprising that the majority of studies, including the most recent publications, referred to TB sequelae without clearly defining their study population. It would therefore be useful to have an overarching term to encompass all post-TB chronic lung conditions. The term PTBLD, as used in this review and used previously by WHO and recent studies, seems to be broad enough to capture all conditions of interest while expressing their distinct and chronic nature. ${ }^{1420}$ The term PTBLD, however, still needs a more precise definition and diagnostic criteria to answer questions such as: Should PTBLD refer to all ex-TB patients with respiratory symptoms and chest X-ray abnormalities? Should PTBLD include, at least, impaired lung function and if so, what should be the cut-off values? Should breathlessness and quality of life be included in the case definition of PTBLD and how should this be assessed? We recommend that an international expert group be convened to discuss the definition and diagnostic criteria of PTBLD.

Principally, the key to prevention and reducing the incidence of PTBLD is early diagnosis and adequate treatment of TB in order to minimise residual lung damage. However, while the incidence of TB may be decreasing (although at a slow rate), there are a considerable number of people in this world with a history of TB (approaching 100 million over the last decade) who are at increased risk of chronic respiratory disorders and should be managed appropriately. This includes those with drug-susceptible and drug-resistant TB disease, and those with comorbidities such as HIV infection and diabetes mellitus. People who are vulnerable to contract $\mathrm{TB}$ are often also those exposed to other risk factors for chronic respiratory diseases, including exposure to household smoke, ambient air pollution and occupational exposure to dust. This makes early identification and management of PTBLD all the more relevant, if we are to halt the rise of chronic respiratory diseases.

\author{
Author affiliations \\ ${ }^{1}$ Clinical Trials and Population Studies Department, University of Plymouth, \\ Plymouth, United Kingdom \\ ${ }^{2}$ Union Headquarters, International Union Against Tuberculosis and Lung Disease, \\ Paris, France \\ ${ }^{3}$ Department of Clinical Research, London School of Hygiene and Tropical Medicine, \\ London, United Kingdom \\ ${ }^{4}$ Department of Pulmonary Medicine and Lung Institute, Makerere University, \\ Kampala, Uganda \\ ${ }^{5}$ Department of Medicine, Therapeutics, Dermatology and Psychiatry, Kenyatta \\ University, Nairobi, Kenya
}

Contributors SCvK, AW and RJ designed the study and the search strategy. AW performed the literature search. SCVK, AW, ME and RJ performed screening. SCVK and ME performed data extraction and labelling. SCvK performed data analysis. BJK, JC, ADH, RJ and SCvK interpreted the results. SCvK wrote the first draft of the manuscript. All authors critically reviewed and approved the final version of the manuscript.

Funding This research was funded in part by the National Institute for Health Research (NIHR) Collaboration for Leadership in Applied Health Research and Care of the South West Peninsula (PenCLAHRC, UK). The paper was funded in part through a grant from La Fondation Veuve Emile Metz-Tesch (Luxembourg).

Disclaimer The views expressed are those of the authors and not necessarily those of the UK's National Health Services (NHS), the NIHR or the Department of Health and Social Care.

Competing interests None declared.

Patient consent Not required.

Provenance and peer review Not commissioned; externally peer reviewed.

Data sharing statement All data used in this study are presented in the manuscript.

Open access This is an open access article distributed in accordance with the Creative Commons Attribution Non Commercial (CC BY-NC 4.0) license, which permits others to distribute, remix, adapt, build upon this work non-commercially, and license their derivative works on different terms, provided the original work is properly cited, appropriate credit is given, any changes made indicated, and the use is non-commercial. See: http://creativecommons.org/licenses/by-nc/4.0/.

\section{REFERENCES}

1. Adeloye D, Chua S, Lee C, et al. Global and regional estimates of COPD prevalence: Systematic review and meta-analysis. J Glob Health 2015;5:020415.

2. Buist AS, McBurnie MA, Vollmer WM, et al. International variation in the prevalence of COPD (The BOLD Study): a population-based prevalence study. Lancet 2007;370:741-50.

3. Soriano JB, Abajobir AA, Abate KH, et al. Global, regional, and national deaths, prevalence, disability-adjusted life years, and years lived with disability for chronic obstructive pulmonary disease and asthma, 1990-2015: a systematic analysis for the Global Burden of Disease Study 2015. Lancet Respir Med 2017;5:691-706.

4. Amaral AF, Coton S, Kato B, et al. Tuberculosis associates with both airflow obstruction and low lung function: BOLD results. Eur Respir J 2015;46:1104-12.

5. Menezes AM, Hallal PC, Perez-Padilla R, et al. Tuberculosis and airflow obstruction: evidence from the PLATINO study in Latin America. Eur Respir J 2007;30:1180-5.

6. Byrne AL, Marais BJ, Mitnick CD, et al. Tuberculosis and chronic respiratory disease: a systematic review. Int $J$ Infect Dis 2015;32:138-46.

7. Allwood BW, Myer L, Bateman ED. A systematic review of the association between pulmonary tuberculosis and the development of chronic airflow obstruction in adults. Respiration 2013;86:76-85.

8. Ehrlich RI, Adams S, Baatjies R, et al. Chronic airflow obstruction and respiratory symptoms following tuberculosis: a review of South African studies. Int J Tuberc Lung Dis 2011;15:886-91.

9. van Zyl Smit RN, Pai M, Yew WW, et al. Global lung health: the colliding epidemics of tuberculosis, tobacco smoking, HIV and COPD. Eur Respir J 2010;35:27-33.

10. Sarkar M, Srinivasa MI, Madabhavi I, et al. Tuberculosis associated chronic obstructive pulmonary disease. Clin Respir J 2017;11:285-95.

11. de la Mora IL, Martínez-Oceguera D, Laniado-Laborín R. Chronic airway obstruction after successful treatment of tuberculosis and its impact on quality of life. Int J Tuberc Lung Dis 2015;19:808-10.

12. Plit ML, Anderson R, Van Rensburg CE, et al. Influence of antimicrobial chemotherapy on spirometric parameters and proinflammatory indices in severe pulmonary tuberculosis. Eur Respir J 1998;12:351-6.

13. Meghji J, Simpson $\mathrm{H}$, Squire SB, et al. A systematic review of the prevalence and pattern of imaging defined post-TB lung disease. PLoS One 2016;11:e0161176.

14. WHO. Global surveillance, prevention and control of chronic respiratory diseases: a comprehensive approach. Geneva: World Health Organization, 2007.

15. WHO. Global tuberculosis report 2017. WHO/HTM/TB/2017.23 ed. Geneva: World Health Organization, 2017. 
16. WHO. Global action plan for the prevention and control of noncommunicable diseases 2013-2020. Geneva: World Health Organization, 2013.

17. WHO. The End TB Strategy. Geneva: World Health Organization, 2015.

18. UN. Transforming our world: the 2030 agenda for sustainable development. New York: United Nations, 2015.

19. WHO. Definitions and reporting framework for tuberculosis -2013 revision. Geneva: World Health Organization, 2013.

20. Chakaya J, Kirenga B, Getahun H. Long term complications after completion of pulmonary tuberculosis treatment: a quest for a public health approach. $J$ Clin Tuberc Other Mycobact Dis 2016;3:10-12.

21. Harries AD, Ade $S$, Burney $P$, et al. Successfully treated but not fit for purpose: paying attention to chronic lung impairment after TB treatment. Int J Tuberc Lung Dis 2016;20:1010-4.

22. Moher D, Liberati A, Tetzlaff J, et al. Preferred reporting items for systematic reviews and meta-analyses: the PRISMA statement. PLoS Med 2009;6:e1000097.

23. Arksey H, O'Malley L. Scoping studies: towards a methodological framework. Int J Soc Res Methodol 2005;8:19-32.

24. Brassey J. Trip. UK: Trip Database Ltd. 2017 https://www. tripdatabase.com/ (accessed 29 Jun 2017).

25. GIN. International Guideline Library. Perthshire: Guidelines International Network. 2016 http://www.g-i-n.net/library/ international-guidelines-library (accessed 29 Jun 2017).

26. Ouzzani M, Hammady H, Fedorowicz Z, et al. Rayyan-a web and mobile app for systematic reviews. Syst Rev 2016;5:210.

27. Australia Government. Guidelines for the control of tuberculosis in the Northern Territory. In: Control CfD. Casuarina: Northern Territory Government, 2016.
28. Malaysia Government. In: Sector TL, ed. Clincal practice guidelines. Managment of drug resistant tuberculosis. 1st edn. Putrajaya: Ministry of Health, 2016

29. Malaysia Government. In: Section M, ed. Management of tuberculosis. 3rd edn. Putrajaya: Ministry of Health, 2012.

30. NICE. NICE guideline: tuberculosis. London: National Institute for Health and Care Excellence, 2016.

31. IUATLD. A tuberculosis guide for specialist physicians. Paris: International Union Against Tuberculosis and Lung Disease, 2003.

32. Spain Government. Clinical practice guideline on the diagnosis, treatment and prevention of tuberculosis. Madrid: Ministry of Science and Innovation, 2010.

33. WHO. Towards tuberculosis elimination: an action framework for low-incidence countries. Geneva: World Health Organization, 2014.

34. Rajeswari R, Muniyandi M, Balasubramanian R, et al. Perceptions of tuberculosis patients about their physical, mental and social wellbeing: a field report from south India. Soc Sci Med 2005;60:1845-53.

35. Hnizdo E, Singh T, Churchyard G. Chronic pulmonary function impairment caused by initial and recurrent pulmonary tuberculosis following treatment. Thorax 2000;55:32-8.

36. Chung KP, Chen JY, Lee $\mathrm{CH}$, et al. Trends and predictors of changes in pulmonary function after treatment for pulmonary tuberculosis. Clinics 2011;66:549-56.

37. Ko Y, Lee YM, Lee HY, et al. Changes in lung function according to disease extent before and after pulmonary tuberculosis. Int J Tuberc Lung Dis 2015;19:589-95.

38. TB Sequel Consortium. TB Sequel. Germany 2017. http://www. tbsequel.org (accessed 29 Jun 2017)

39. Jones R, Kirenga BJ, Katagira W, et al. A pre-post intervention study of pulmonary rehabilitation for adults with post-tuberculosis lung disease in Uganda. Int $J$ Chron Obstruct Pulmon Dis 2017;12:3533-9. 